CERN-TH/2002-256

\title{
Rotating Strings in Confining AdS/CFT Backgrounds
}

\author{
A. Armoni, J. L. F. Barbón ${ }^{1}$ and A. C. Petkou \\ Theory Division, CERN \\ CH-1211 Geneva 23, Switzerland \\ adi.armoni@cern.ch,barbon@cern.ch,tassos.petkou@cern.ch
}

\begin{abstract}
We study semiclassical rotating strings in AdS/CFT backgrounds that exhibit both confinement and finite-size effects. The energy versus spin dispersion relation for short strings is the expected Regge trajectory behaviour, with the same string tension as is measured by the Wilson loop. Long strings probe the interplay between confinement and finite-size effects. In particular, the dispersion relation for long strings shows a characteristic dependence on the string tension and the finite-size scale.
\end{abstract}

${ }^{1}$ On leave from Departamento de Física de Partículas da Universidade de Santiago de Compostela, Spain. 


\section{Introduction}

The study of AdS/CFT duality (see [1, 2, 3, 4]) beyond the BPS sector has benefited mostly from the semiclassical approximation. Any approach of this type is based on two main dynamical assumptions: first, we assume that the AdS-like background that describes the vacuum can be studied in the supergravity approximation. Typically, this involves some tunable dimensionless number that controls the $\alpha^{\prime}$ corrections in the gravity description. For the standard example of $\mathrm{AdS}_{5} \times \mathbf{S}^{5}$ with radius $R=\sqrt{\alpha^{\prime}}\left(4 \pi g_{s} N\right)^{1 / 4}$, this quantity is $\alpha^{\prime} / R^{2}=\lambda^{-1 / 2}$, with $\lambda=g_{\mathrm{YM}}^{2} N$ the 't Hooft coupling of the $\mathcal{N}=4$ super Yang-Mills (SYM) theory. This type of condition is common to all AdS/CFT examples and represents the main obstacle in the application of AdS/CFT ideas to realistic gauge theories such as QCD.

The second condition of the semiclassical approximation is that the excitation itself be quasiclassical, in the sense that it can be described as a solitonic object. This requires some large quantum number that may or may not be of topological nature [5]. Typical examples are the various holographic interpretations of branes in AdS as instantons, Wilson lines, domain walls, etc. Another example is the interpretation of the large AdS black hole as a canonical thermal ensemble in the CFT. Most of these examples involve either instantons or static solitonic objects.

Recently, Gubser, Klebanov and Polyakov (GKP) have studied some instances of stationary solitons of periodic type [6]. One particular example where one can go much beyond the semiclassical approximation is that of highly boosted point-like strings, corresponding to states of large $R$-charge [7]. Other examples include truly extended classical string states, such as folded strings in the leading Regge trajectory, stabilized by a large spin $S$, and/or large $R$-charge quantum number $J$ (see also [8]-[13] and [14] for an earlier work.).

In particular, for very large rotating strings in $\mathrm{AdS}_{5}$ in global coordinates, there is a dispersion relation

$$
E R=S+\frac{\sqrt{\lambda}}{\pi} \log S
$$

valid for $S \gg \sqrt{\lambda} \gg 1$. This "rotor" represents a high-spin state in the dual CFT defined on a three-sphere $\mathbf{S}^{3}$ of radius $R$. It is stable in the large- $N$ limit and, by the state/operator map, it corresponds to a local operator on $\mathbf{R}^{4}$. This operator has spin $S$ with respect to the $S O(4)$ group that leaves the insertion point fixed in $\mathbf{R}^{4}$ and anomalous dimension

$$
\Delta=E R=S+\frac{\sqrt{\lambda}}{\pi} \log S .
$$

This is a very interesting prediction that can be compared with similar results for the anoma-

lous dimensions of twist-two operators for large spin in weakly-coupled gauge theories such as realistic QCD $[15,16,17]$. 
In a previous paper [10], we have shown that the dispersion relation (1) applies also in the high-temperature plasma phase, but only for states of spin $S \gg \sqrt{\lambda}(R T)^{4}$. This is in agreement with the expected melting of glueballs when their energy approaches the average plasma kinetic energy. In the AdS picture, they correspond to "planetoids" [18, 19] orbiting the large AdS black hole that represents the thermal ensemble.

In this note we study generalizations of the GKP proposal [6] to backgrounds that describe confining theories. The appropriate generalization of Eq. (2) is far from straightforward, because there is no exact one-to-one state/operator map for non-conformal theories. It was suggested in [6] that non-periodic solitons should be considered in time-dependent backgrounds (see also [20]).

We choose to discuss only dispersion relations of stationary states and postpone the discussion of what the implications are (if any) for the spectrum of anomalous dimensions of local operators. Since we are interested in matching the dispersion relation (1) at large spin we are led to the consideration of AdS models in global coordinates, which represent theories in finite volume. In section 2 we discuss some general aspects of the interplay between confinement and finite-size effects in the context of semiclassical AdS/CFT. In particular, in subsection 2.1 we consider a toy model that illustrates these issues. In section 3 we study similar features of semiclassical string states with large flavour charge in the standard confining model of Witten [21, 22]. Section 4 is devoted to a discussion.

\section{Confinement vs. Finite-Size Effects}

In a large- $N$ confining theory in infinite volume where glueballs are described by closedstring states, the obvious candidate for a high-spin soliton is a fast-spinning glueball in the leading Regge trajectory. In the large- $N$ limit, it should correspond to a folded closed

spinning configuration of the confining string. Hence, the classic Regge dispersion relation in flat space,

$$
E=\sqrt{4 \pi \sigma S}
$$

is a characteristic feature of the glueball spectrum of any confining theory in the large- $N$ limit. In (3) the constant $\sigma$ is the string tension, the same as can be measured via the static potential between heavy quark sources.

It is straightforward to find the required soliton in any AdS/CFT model of confinement. For a gauge theory in non-compact $\mathbf{R}^{d}$, the gravity description involves, among other things, a warped metric

$$
d s^{2}=f(z) d s^{2}\left(\mathbf{R}^{d}\right)+d z^{2}+\ldots
$$

where $d s^{2}\left(\mathbf{R}^{d}\right)$ is the standard Minkowski metric on $\mathbf{R}^{d}$, the variable $z$ represents the holographic coordinate and the dots stand for internal factors of the full string background. 
Confinement arises when $f(z)$ has a local minimum at $z_{*}$ with $f\left(z_{*}\right)>0$. In many examples, spacetime is literally restricted to $z>z_{*}$. In this situation one has an effective gravitational potential that stabilizes string configurations at fixed $z=z_{*}$ and extended in $\mathbf{R}^{d}$. Hence, Wilson lines saturate at $z_{*}$ with effective string tension [23, 24, 25]

$$
\sigma=\frac{f\left(z_{*}\right)}{2 \pi \alpha^{\prime}}
$$

By the same token, folded closed strings that rotate in $\mathbf{R}^{d}$ will be stabilized at $z=z_{*}$ and satisfy (3) with string tension (5).

An intriguing result of [6] is that semiclassical Regge trajectories of type (3) can be found in the spectrum of an exact CFT. For spins in the range $1 \ll S \ll \sqrt{\lambda}$ the folded strings rotate deep inside AdS and do not feel the curvature. Then one finds the law (3) with string tension

$$
2 \pi \sigma_{\mathrm{CFT}}=\frac{1}{\alpha^{\prime}}=\frac{\sqrt{\lambda}}{R^{2}} .
$$

Of course, this is not surprising in itself, given that the model does contain strings. However, in this case the "string tension" (6) is tied to the scale of finite-size effects (the size $R$ of the $\mathbf{S}^{3}$ ), and it cannot be probed by static quark sources because the corresponding quarkantiquark distance cannot be larger than $\mathcal{O}(R)$.

Hence, we see that standard Regge trajectories can be the landmark of true confining phenomena, but they can also appear as artefacts of finite-size effects at strong coupling. In fact, in the GKP description of the $\mathcal{N}=4 \mathrm{SYM}$ model, both the Regge trajectories and the logarithmic correction in (1) arise as peculiar finite-size effects.

It would be interesting to see if true confining Regge trajectories can coexist with a logarithmic high-spin asymptotics as in Eq. (1). For this purpose, one should consider a confining theory defined on a spatial 3-sphere of finite radius $R$, with an intrinsic glueball mass gap $\Lambda_{\mathrm{QCD}} \gg 1 / R$, so that confining Regge trajectories can be identified independently of the finite-size effects. If the high-energy asymptotics, $E \gg \Lambda_{\mathrm{QCD}} \gg 1 / R$ is well approximated by a conformal theory, then one should also find (1) for very high spin.

Unfortunately, all existing AdS/CFT models of confinement have the form (4) and hence describe gauge theories in infinite volume. In order to exhibit a family of stationary periodic strings interpolating between confining Regge trajectories and (1), one must construct a background that is asymptotic to $\mathrm{AdS}_{5}$ space in global coordinates,

$$
d s^{2} \approx-\left(1+r^{2} / R^{2}\right) d t^{2}+\frac{d r^{2}}{1+r^{2} / R^{2}}+r^{2} d \Omega_{3}^{2}+\ldots,
$$

as $r \rightarrow \infty$, but shows a "confinement sphere" at some intermediate radius $r=r_{*} \gg R$. By a confinement sphere we mean a region with a repulsive gravitational potential for test masses. Under these conditions, static strings that represents interacting quark-antiquark 


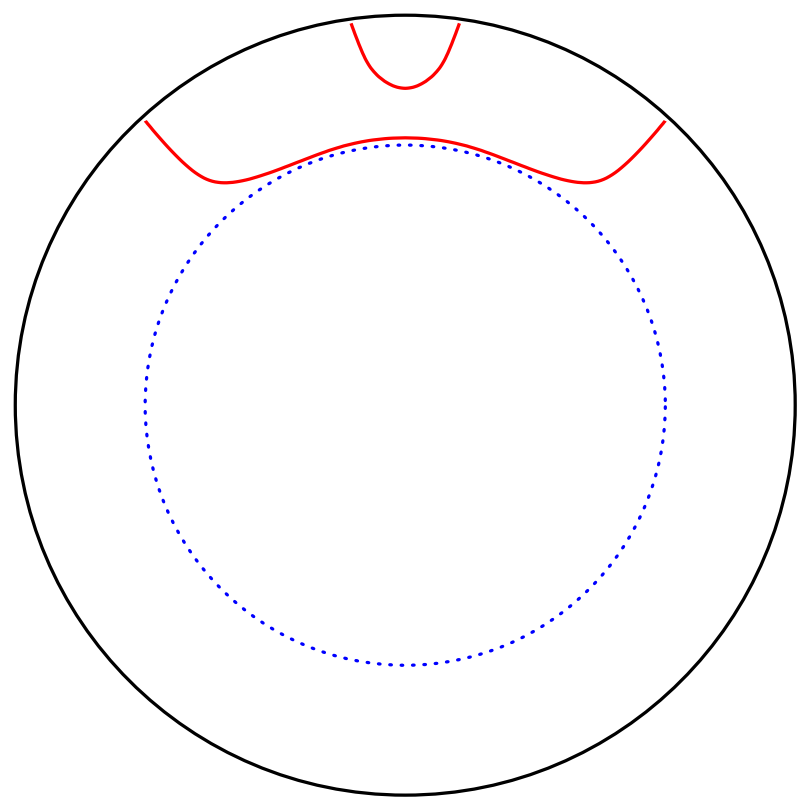

Figure 1: Behaviour of Wilson lines in a model of confinement in finite volume. The dotted line denotes the "confining sphere" at $r=r_{*}$. Strings that correspond to quark-antiquark pairs that are sufficiently separated in the 3 -sphere will saturate there.

pairs would behave as in fig. 1. A static string subtending an angle $\theta \ll \pi$ in the angular $\mathbf{S}^{3}$ drops to the interior of AdS down to a radius of order

$$
r_{\min } \sim \frac{R}{\theta} .
$$

However, when $r_{\min } \sim r_{*}$, the repulsive gravitational potential stabilizes the string as in fig. 1. After the standard subtraction of the infinite "bare quark mass", one is left with a contribution to the interaction energy that grows linearly with the proper length on the spatial $\mathbf{S}^{3}$, i.e. a confining static potential.

It is clear from the picture that short folded strings, rotating in a plane tangent to the 3-sphere at $r=r_{*}$, will be stabilized from falling to smaller $r$, just like the static strings are. If the length of the rotor is small with respect to the radius of the confining sphere, the dispersion relation will be approximately Regge-like, $E \sim \sqrt{S}$, with some effective string tension. On the other hand, for very large spin, the rotor is much larger than the confining sphere, and its properties are well approximated by the rotor in vacuum AdS space (7). In this way we describe a family of periodic solitons that interpolate between confining Regge trajectories and the logarithmic behaviour (1). 


\subsection{A Toy Model of Confinement in Finite Volume}

It would be very interesting to exhibit a consistent AdS/CFT model with the qualitative features shown in fig. 1. Lacking such a model, we introduce in this section a formal toy model with the required properties. It can be constructed as an unphysical limit of an R-charge condensate of $\mathcal{N}=4 \mathrm{SYM}$ on $\mathbf{S}^{3}$. The AdS/CFT representation of a thermal ensemble with such non-vanishing charge $Q$ is in terms of an AdS charged black hole with metric [26]

$$
d s^{2}=-f(r) d t^{2}+f^{-1}(r) d r^{2}+r^{2} d \Omega^{2}
$$

with

$$
f(r)=1+r^{2}-\frac{M}{r^{2}}+\frac{Q^{2}}{r^{4}} .
$$

Note that the metric is asymptotic to AdS in global coordinates, with units chosen so that $R=R_{\mathrm{AdS}}=1$. For large values of $M / Q$ the equation $f(r)=0$ has two positive roots, the largest one being the horizon of the black hole. In the extremal limit the two roots coalesce into a single one. At this point the black hole temperature vanishes and the solution represents a zero-temperature charge condensate. The associated extremal mass $M$ gives the energy cost of such a condensate. If we now "overcharge" the black hole beyond the extremal limit, the function $f(r)$ becomes positive and develops a minimum at some positive radius $r_{*}$.

Since $f(r)$ is a gravitational potential for static masses at fixed $r$, the 3-sphere at $r=r_{*}$ satisfies the conditions for a "confinement wall", in the sense of the previous section. On the other hand, the singularity at $r=0$ becomes naked and presumably unphysical (i.e. not resolved by stringy effects). In order to simplify the formulas, we can take the extreme situation with $M=0$. Then $r_{*} \sim Q^{1 / 3}$ and $r_{*} \gg R_{\text {AdS }}$ for $Q \gg 1$. In this regime, the region of the geometry that is responsible for the confinement properties is well separated from the naked singularity.

Hence, we have a geometry that simulates a confining behaviour in finite volume, in such a way that the confining length scale is well contained inside the box. We use this setup simply as a formal model, since the original condensate in the $\mathcal{N}=4 \mathrm{SYM}$ theory is rather unphysical for $M / Q \rightarrow 0$. We think, nevertheless, that any consistent gravity description of a similar confining theory in finite volume should have the generic properties exhibited by this model.

Semiclassical rotors of proper length much smaller than $r_{*}$ stabilize in a plane tangent

to the "confining sphere" $r=r_{*}$, as in fig. 2. Let us pick a north pole for this sphere and single out a two-dimensional subspace of the tangent space at this north pole, with polar coordinates $(\rho, \phi)$. Then, in the vicinity of this point, the relevant $(2+1)$-dimensional metric 
is approximately flat:

$$
d s^{2} \approx f\left(r_{*}\right)\left(-d t^{2}+d \rho^{2}+\rho^{2} d \phi^{2}\right)
$$

Hence, there is an approximate Regge trajectory for small strings stabilized around the north pole with the dispersion relation

$$
E \approx \sqrt{4 \pi \sigma S}, \quad \sigma=\frac{f\left(r_{*}\right)}{2 \pi \alpha^{\prime}} \sim \frac{Q^{2 / 3}}{\alpha^{\prime}} .
$$

As the spin grows, these rotors start feeling the curved background geometry; they will follow the dashed line in fig. 2 and eventually their proper length becomes much larger than $r_{*}$. At this point we can approximate the dispersion relation of such "eccentric" rotors by that of the central rotors that pass through the singularity.

A folded string rotating on an equator of $\mathbf{S}^{3}$ with angular velocity $\omega$ has action

$$
I_{\mathrm{NG}}=-\frac{2}{\pi \alpha^{\prime}} \int d t d r \sqrt{1-\frac{\omega^{2} r^{2}}{f(r)}} .
$$

Therefore, it will be subluminal if

$$
\omega^{2} r^{2} \leq f(r)
$$

with the equality determining the folding points of the string. We see that, in the limit that $M \rightarrow 0$, this equation has solutions of finite energy only for $\omega>1$, and they correspond to rotors that extend from the naked singularity at $r=0$ up to the turning point $r_{\max }$.

For $Q \gg 1$, there are three regimes of interest. First, we have short strings with $r_{\max } \ll Q^{1 / 3}$. These are presumably irrelevant to our purposes, since their properties are characteristic of the region dominated by the singularity. It is interesting, however, to notice that both the energy and the spin of such strings are finite. A simple scaling argument yields the law (see Appendix A for an explicit calculation) $E^{4} \sim Q S / \alpha^{\prime 3}$.

Conversely, for very long rotors we expect the dispersion relation to approach that of standard AdS space, since most of the string is rotating far from the "confining" region. So, we obtain

$$
E \sim S+\frac{\sqrt{\lambda}}{\pi} \log S
$$

asymptotically for very large spin.

The interesting feature of this model is that in addition to the two above asymptotic regimes there exists an intermediate one where the rotor endpoints are in the range:

$$
Q^{1 / 3} \ll r_{\max } \ll Q^{1 / 2} .
$$

In Appendix A we prove that in this regime the rotors obey the following law

$$
E R-S \approx A\left(1-c(A / S)^{1 / 2}\right)
$$




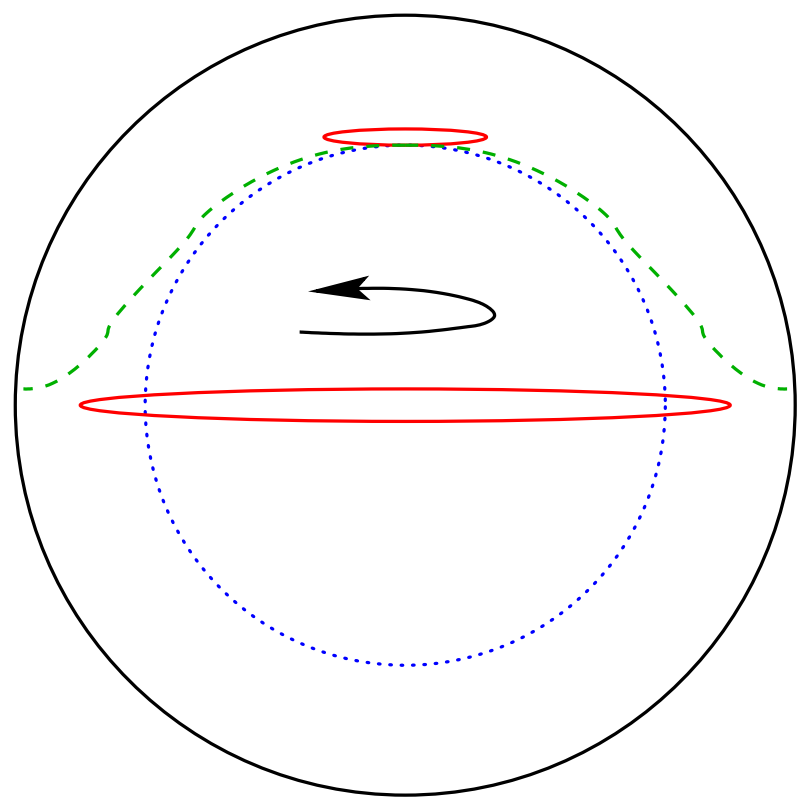

Figure 2: Rotors in the charged black hole model. The dotted sphere denotes the radius $r=r_{*} \sim$ $Q^{\frac{1}{3}}$. The "eccentric" rotors sit at $r=r_{*}$, whereas the "central" rotors pass through $r=0$.

where we have restored the radius of the sphere by dimensional analysis. The constant $c$ is a positive numerical coefficient of $\mathcal{O}(1)$ and

$$
A \propto \frac{Q^{1 / 3} R^{2}}{\alpha^{\prime}} \sim \lambda^{1 / 4} \sqrt{\sigma R^{2}} .
$$

Therefore, we arrive at the following picture. The flat-space Regge behaviour characteristic of confinement extends for spins in the range $1 \ll S \ll \sqrt{\lambda} Q^{\frac{1}{3}}$. At approximately the upper limit there is a crossover to the particle-like relativistic behaviour (14). This intermediate regime extends in the range of spins

$$
\sqrt{\lambda} Q^{\frac{1}{3}} \ll S \ll \sqrt{\lambda} Q
$$

where the upper limit signals the final crossover to the logarithmically corrected relativistic behaviour that is characteristic of the conformal theory on $\mathbf{S}^{3}$.

The rotors can be considered as probes of the peculiar interplay between confinement and finite-size effects in these models. One way of expressing this interplay in graphical terms is to define a notion of effective length of the string in terms of gauge-theory quantities. One possible definition would be in terms of the effective world-sheet area spanned in one period of motion, equal to $2 \pi / \omega$. That is, we write the Nambu-Goto action as

$$
2 \pi \alpha^{\prime} I_{\mathrm{NG}}=-\frac{2 \pi}{\omega} \cdot L_{\mathrm{eff}}
$$


The String Effective Length

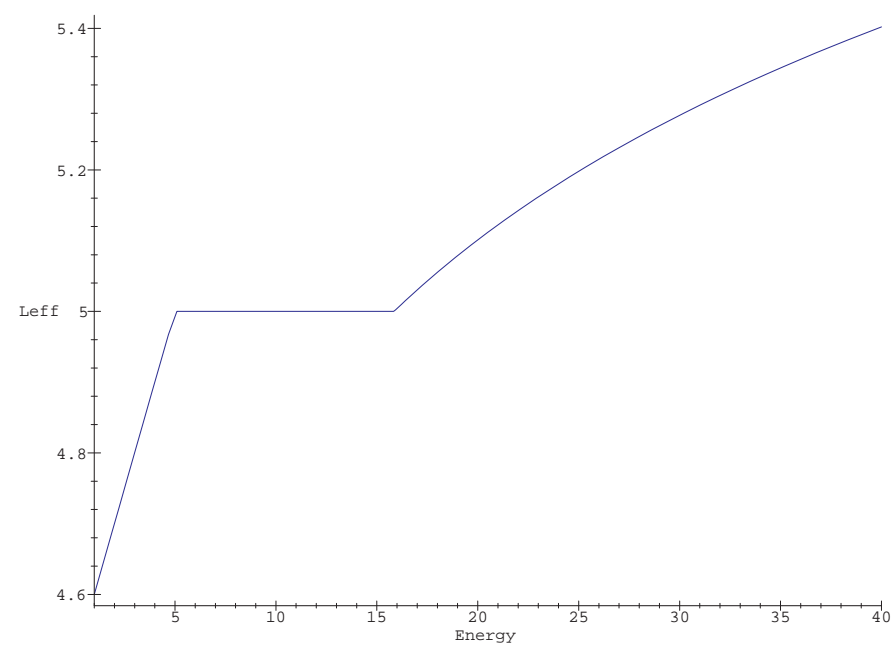

Figure 3: The effective length of the string as a function of energy. For energies lower than $Q^{\frac{1}{3}} / R$ (short strings) the length grows linearly. Finite-size effects induce a transient plateau where the string length is locked to the size of the box. For larger energies this plateau tilts with the logarithmic growth characteristic of the conformal fixed point.

With this definition, $L_{\text {eff }}$ measures the correction to the ultrarelativistic dispersion relation $E R=S$, i.e. we have

$$
L_{\mathrm{eff}}=2 \pi \alpha^{\prime}(E-\omega S) .
$$

A confining Regge trajectory is characterized by strings that grow linearly with energy

$$
L_{\text {eff }}(E) \propto E .
$$

On the other hand, the subleading logarithm of conformal theories on $\mathbf{S}^{3}$ gives an anomalous logarithmic growth of the string $L_{\text {eff }} \propto \log (E)$ in this regime. The intermediate regime (16) is associated to a saturation effect on the length of the string, which stays "locked" on the size of the box

$$
L_{\mathrm{eff}} \approx R, \quad \text { for } \quad \sqrt{\lambda} Q^{\frac{1}{3}} \ll S \ll \sqrt{\lambda} Q .
$$

We illustrate this behaviour in fig. 3 .

Hence, we see that the spectroscopy of high-spin glueballs in these models reveals the finite-size effects in the high energy regime, in yet another interesting manifestation of UV/IR effects for extended objects. 


\section{Rotating Strings in Witten's Model of Confinement}

An entirely different aspect of the interplay between confinement and finite-size effects is provided by the models in refs. [21, 22]. In this case, one starts from a non-confining SYM theory on $\mathbf{S}^{1} \times \mathbf{R}^{p}$, with supersymmetry-breaking boundary conditions on the circle of radius $R$. In the limit of dimensional reduction, one should recover ordinary non-supersymmetric Yang-Mills theory on $\mathbf{R}^{p}$. Thus, supersymmetry-breaking by finite-size effects in a higher dimensional theory induces standard confinement (in infinite volume) after dimensional reduction. The purpose of this section is to use the semiclassical rotating strings as probes of this mechanism.

A large- $N$ gravity description of these models is obtained by looking at the metric of near-extremal D $p$ branes [27]

$$
d s^{2}=r^{\frac{7-p}{2}}\left(R^{2} h(r) d \phi^{2}+d \vec{y}^{2}-d t^{2}\right)+r^{\frac{p-7}{2}} \frac{d r^{2}}{h(r)}+\frac{r^{2}}{r^{\frac{7-p}{2}}} d \Omega_{8-p}^{2},
$$

where the function $h(r)$ is given by

$$
h(r)=1-\left(\frac{r_{0}}{r}\right)^{7-p}
$$

and we use units such that the charge radius of the $\mathrm{D} p$-brane is set to unity: $1=R_{q} \sim$

$\sqrt{\alpha^{\prime}}\left(g_{s} N\right)^{\frac{1}{7-p}}$. The supergravity approximation to this background is good when the 't Hooft coupling of the $(p+1)$-dimensional SYM theory is large at the scale of the compact circle, i.e. when

$$
g_{p+1}^{2} N R^{p-3} \gg 1
$$

The metric is smooth at $r=r_{0}$ if $r_{0}$ is fixed as a function of $R$ via

$$
R=\frac{2}{7-p} r_{0}^{\frac{p-5}{2}}
$$

Hence, space-time terminates at $r=r_{0}$, which behaves as a "confining wall" in this model. The associated string tension, as measured by static quark sources separated in $\mathbf{R}^{p}$ is

$$
\sigma=\frac{r_{0}^{\frac{7-p}{2}}}{2 \pi \alpha^{\prime}}
$$

Hence, folded closed strings sitting at $r=r_{0}$ and rotating in a plane contained in $\mathbf{R}^{p}$ will follow a Regge dispersion relation $E=\sqrt{4 \pi \sigma S}$. These rotors are unaffected by finite-size effects at high energy because $\mathbf{R}^{p}$ is non-compact.

The interesting feature of this model is that stable rotors also occur along the "cigar" factor of the metric, namely the $(r, \phi)$ space that fills the $\mathbf{S}^{1}$ circle in the bulk supergravity background. These rotors have constant angular velocity in the $\mathbf{S}^{1}$ circle of radius $R$, i.e. 
$\phi=\omega t$. The associated angular momentum is nothing but the linear momentum along the compact circle. We shall denote its quantum by $n$.

The action of these solitons is given by

$$
I_{\mathrm{NG}}=-\frac{2}{\pi \alpha^{\prime}} \int d t d r \sqrt{\frac{1}{h(r)}-\omega^{2} R^{2}},
$$

where the range of the radial variable is $r_{0} \leq r \leq r_{\max }$. The turning point is given by the solution of

$$
\omega^{2} R^{2}=\frac{1}{h\left(r_{\max }\right)},
$$

so that $\omega R$ approaches unity from above when $r_{\max } \rightarrow \infty$. The expressions for the energy and quantized compact momentum of these solitons are

$$
\begin{aligned}
& E=\frac{2}{\pi \alpha^{\prime}} \int_{r_{0}}^{r_{\max }} \frac{d r}{h(r)} \frac{1}{\sqrt{h(r)^{-1}-\omega^{2} R^{2}}} \\
& n=\frac{2}{\pi \alpha^{\prime}} \int_{r_{0}}^{r_{\max }} d r \frac{\omega R^{2}}{\sqrt{h(r)^{-1}-\omega^{2} R^{2}}}
\end{aligned}
$$

These integrals are reducible to standard hypergeometric functions, which we present in Appendix B.

There are two interesting regimes. For $r_{\max } \ll R$ we have short rotors in locally flat space near the tip of the cigar at $r \approx r_{0}$. These rotors follow linear Regge trajectories of the form

$$
E=\sqrt{4 \pi \sigma n}
$$

The second regime corresponds to very long rotors $r_{\max } \gg R$ that "climb up" the cigar towards the boundary. The corresponding dispersion relation is

$$
E R=n+b \sigma R^{2}\left[1-c\left(\frac{\sigma R^{2}}{n}\right)^{\nu}\right],
$$

with $b, c$ positive numerical constants and $\nu=(5-p) /(9-p)$. Hence, we find the same saturation behaviour as in the previous model. The effective string length $L_{\text {eff }}$ grows linearly with the energy up to the crossover momenta of order $n \sim \sigma R^{2}$. At these values of the momentum it levels off to a plateau that continues for arbitrarily high energies; the "finitesize locking".

The physics of the long "cigar rotors" in this model is rather interesting when compared with the supersymmetric counterpart corresponding to the same $\mathbf{S}^{1} \times \mathbf{R}^{p}$ space-time with supersymmetric boundary conditions on the circle. In that case the circle remains noncontractible in the bulk, since $r_{0}=0$. The cigar becomes a cylinder and string states of momentum $n$ are point-like in the classical approximation. The corresponding dispersion relation is $E=n$, up to corrections of order 1 in string units. 
The nonsupersymmetric model behaves very differently. By looking at the $n \rightarrow \infty$ asymptotics, we would expect to probe just the short-distance behaviour of the supersymmetric $(p+1)$-dimensional theory. However, we see that solitonic objects remain at any arbitrarily large momentum in such a way that their wave function has a non-trivial overlap with all scales down to the confining scale $1 / R$. The leading deviation from the supersymmetric dispersion relation is semiclassical and depends on the string tension of the low-energy confining theory.

\section{Discussion}

One of the most striking aspects of AdS/CFT models in the strong coupling limit is the emergence of higher-dimensional bulk physics, codified in the Hilbert space of the fourdimensional CFT. The prime example of this phenomenon is the density of states of $\mathcal{N}=4$ SYM on $\mathbf{S}^{3}$ (see [4] for a summary). For $N \gg \lambda \gg 1$ a large hierarchy of regimes opens up between the naive mass gap $E \sim 1 / R$ and the large- $N$ phase transition threshold $E \sim N^{2} / R$. For example, in the range $1 / R \ll E \ll \lambda^{1 / 4} / R$ the density of states is well approximated by a massless ten-dimensional gas. At the upper limit the density of states turns into a Hagedorn spectrum of ten-dimensional strings with tension of order $1 / \alpha^{\prime} \sim \sqrt{\lambda} / R^{2}$.

One can interpret the GKP results in a similar vein. In the Hamiltonian interpretation we are looking at the spectrum of very particular states on $\mathbf{S}^{3}$, namely single-trace (glueball) states with maximal spin for a given energy. In the gravity dual they correspond to classical rotating folded strings. In view of the previous considerations regarding the density of states, it is natural that we find a ten-dimensional Regge trajectory with "string tension" $\sigma_{\mathrm{CFT}} \sim \sqrt{\lambda} / R^{2}$ in the range of energies $\lambda^{1 / 4} / R \ll E \ll \lambda^{1 / 2} / R$. However, it is also clear that in this model the string tension is an artefact of the finite-size effects. At very high energies the dispersion relation reproduces an anomalous logarithmic growth of the spinning "glueball".

In this paper we have studied GKP-type periodic solitons in models where true confinement phenomena coexist with finite-volume constraints. We identify semiclassical rotors representing glueball states on Regge trajectories $E=\sqrt{4 \pi \sigma S}$, where $\sigma$ is a true string tension that would survive in the infinite-volume limit. Our main result is the identification of a "saturation" effect caused by the finite volume. Namely, the linear growth of the effective string size along the Regge trajectory reaches a plateau when the glueball hits the walls of the box.

Our interpretation of the dispersion relations in various examples suggests that this saturation is a generic effect induced by the finite size of either the physical space or the internal space associated with a conserved charge ( $R$-charge or compact momentum). 
In models whose extreme high-energy behaviour is well approximated by a CFT on $\mathbf{S}^{3}$, we find that the effective size of the glueball ends up showing the logarithmic growth of GKP. Thus, the existence of the transient plateau in these models is really a consequence of the hierarchy $\sigma \gg \sigma_{\mathrm{CFT}}$, between the true confining string tension and the artificial string tension of an exact CFT at strong coupling.

Coming from the high-energy side, we could say that the flattening of the CFT logarithm is the signal of confinement at low energy. We regard this as an interesting speculation, because the logarithm itself is visible in ordinary perturbation theory in realistic QCD. At weak coupling, it arises from gluon-exchange effects in the computation of anomalous dimensions. Therefore, it is tempting to regard the plateau as the onset of confinement effects in the sense that "gluon effects" become better described as "glueball effects".

The main obstacle in the development of these ideas is the lack of an exact correspondence between energies on $\mathbf{S}^{3}$ and conformal dimensions on $\mathbf{R}^{4}$, once we are away from the conformal fixed point. It would be very interesting to find the appropriate generalization of this fact that is useful to our discussion of confining models.

\section{ACKNOWLEDGEMENTS}

A.A. would like to thank A. Font, J. Sonnenschein and S. Theisen for useful discussions. A.C.P. wishes to thank E. Floratos for interesting remarks.

\section{Appendices}

\section{A Dispersion relations for rotating strings in the charged AdS black hole}

We study rotating strings in the background (8),(9) (with $M=0, Q^{2} \gg 1$ ). We use the prescription of [6]. The string configuration extends in the radial $r$ direction of space-time and rotates along the $\phi$ angle with constant angular velocity $\dot{\phi}=\omega$. The Nambu-Goto action

$$
I_{\mathrm{NG}}=-\frac{1}{2 \pi \alpha^{\prime}} \int \mathrm{d} \tau \mathrm{d} \sigma \sqrt{-\operatorname{det} G_{\mu \nu} \partial_{\alpha} X^{\mu} \partial_{\beta} X^{\nu}}
$$

takes the following form

$$
I_{\mathrm{NG}}=-\frac{1}{2 \pi \alpha^{\prime}} \int \mathrm{d} \tau \int \mathrm{d} \sigma \sqrt{-G_{r r}\left(\frac{d r}{d \sigma}\right)^{2}\left(G_{t t}+G_{\phi \phi} \dot{\phi}^{2}\right)} .
$$


For a metric of the form (8), with (9) it yields

$$
I_{\mathrm{NG}}=-\frac{4}{2 \pi \alpha^{\prime}} \int \mathrm{d} \tau \int_{0}^{r_{\max }} \mathrm{d} r \sqrt{1-\dot{\phi}^{2} \frac{r^{2}}{f(r)}}=-\frac{4 \tau}{2 \pi \alpha^{\prime}} \int_{0}^{r_{\max }} \mathrm{d} r \sqrt{1-\dot{\phi}^{2} \frac{r^{2}}{1+r^{2}+Q^{2} / r^{4}}} .
$$

The integration range $0 \leq r \leq r_{\max }$ is determined by the condition that the square root in (31) be real, namely by the requirement

$$
1+r^{2}+\frac{Q^{2}}{r^{4}} \geq \omega^{2} r^{2}
$$

The energy and angular momentum of the string are then given by

$$
\begin{aligned}
& E=\frac{2}{\pi \alpha^{\prime}} \int_{0}^{r_{\max }} \mathrm{d} r \frac{1}{\sqrt{1-\omega^{2} \frac{r^{2}}{f(r)}}}=\frac{2}{\pi \alpha^{\prime}} \int_{0}^{r_{\max }} \mathrm{d} r \sqrt{\frac{r^{4}+r^{6}+Q^{2}}{r^{4}+\left(1-\omega^{2}\right) r^{6}+Q^{2}},} \\
& S=\frac{2}{\pi \alpha^{\prime}} \int_{0}^{r_{\max }} \mathrm{d} r \frac{\omega \frac{r^{2}}{f(r)}}{\sqrt{1-\omega^{2} \frac{r^{2}}{f(r)}}}=\frac{2}{\pi \alpha^{\prime}} \int_{0}^{r_{\max }} \mathrm{d} r \frac{\omega r^{6}}{\sqrt{\left(r^{4}+r^{6}+Q^{2}\right)\left(r^{4}+\left(1-\omega^{2}\right) r^{6}+Q^{2}\right)}}
\end{aligned}
$$

The condition for real roots, eq.(32) leads to three interesting regimes

$$
\begin{array}{ccl}
\text { (i). } & r_{\max } \ll Q^{\frac{1}{3}} & \text { Short strings. } \\
\text { (ii). } & Q^{\frac{1}{3}} \ll r_{\max } \ll Q^{\frac{1}{2}} & \text { Intermediate regime. } \\
\text { (iii). } & Q^{\frac{1}{2}} \ll r_{\max } & \text { Long strings. }
\end{array}
$$

\section{A.1 Short strings}

Let us consider first the region (35). When the strings are very short, one can choose a very fast rotation, such that $\omega^{2} r_{\max }^{6}=Q^{2}$, for a given fixed $Q$. For this case

$$
\begin{aligned}
& E \sim \frac{1}{\alpha^{\prime}}\left(\frac{Q}{\omega}\right)^{\frac{1}{3}}, \\
& S \sim \frac{1}{\alpha^{\prime}}\left(\frac{Q}{\omega}\right)^{\frac{1}{3}} \frac{1}{\omega} .
\end{aligned}
$$

Note that although we have a singularity at the origin, both the energy and the spin do not diverge. For the short string we obtain the relation

$$
E^{4} \sim \frac{Q}{\alpha^{\prime 3}} S .
$$




\section{A.2 Intermediate regime}

Next, consider strings in the regime

$$
r_{\max } \ll Q^{\frac{1}{2}}
$$

In this regime we can approximate the energy (33) and angular momentum (34) by

$$
\begin{aligned}
E & =\frac{2}{\pi \alpha^{\prime}} \int_{0}^{r_{\max }} \mathrm{d} r\left(1+\frac{1}{Q^{2}} r^{6}\right)^{\frac{1}{2}}\left(1-\frac{\eta}{Q^{2}} r^{6}\right)^{-\frac{1}{2}} \\
S & =\frac{\omega}{Q^{2}} \frac{2}{\pi \alpha^{\prime}} \int_{0}^{r_{\max }} \mathrm{d} r r^{6}\left(1+\frac{1}{Q^{2}} r^{6}\right)^{-\frac{1}{2}}\left(1-\frac{\eta}{Q^{2}} r^{6}\right)^{-\frac{1}{2}} .
\end{aligned}
$$

Now, the parameter $\eta=\omega^{2}-1$ determines the "length" of the strings since

$$
r_{\max } \approx \frac{Q^{\frac{1}{3}}}{\eta^{\frac{1}{6}}} .
$$

In particular, as $\eta \rightarrow 0$ we have $r_{\max } \gg Q^{1 / 3}$. Therefore, under the assumption that $Q$ is very large, we can consider "long strings" in the regime

$$
Q^{\frac{1}{3}} \ll r_{\max } \ll Q^{\frac{1}{2}}
$$

The integrals (42) and (43) can be explicitly evaluated with the results:

$$
\begin{aligned}
& E=\frac{Q^{\frac{1}{3}}}{6} \frac{2}{\pi \alpha^{\prime}} \frac{1}{(1+\eta)^{\frac{1}{6}}} B\left(\frac{1}{2}, \frac{1}{6}\right){ }_{2} F_{1}\left(\frac{1}{6}, \frac{7}{6} ; \frac{2}{3} ; \frac{1}{1+\eta}\right), \\
& S=\frac{Q^{\frac{1}{3}}}{6} \frac{2 \omega}{\pi \alpha^{\prime}} \frac{1}{(1+\eta)^{\frac{7}{6}}} B\left(\frac{1}{2}, \frac{7}{6}\right){ }_{2} F_{1}\left(\frac{7}{6}, \frac{7}{6} ; \frac{5}{3} ; \frac{1}{1+\eta}\right) .
\end{aligned}
$$

It is interesting to note that despite the fact that both expressions above diverge as $\eta \rightarrow 0$, they never exhibit logarithmic singularities. Furthermore, the divergences cancel exactly in the difference $E-S$ and the result is

$$
\begin{aligned}
E-S & =\frac{Q^{\frac{1}{3}}}{6} \frac{2}{\pi \alpha^{\prime}}\left[\frac{\Gamma\left(\frac{1}{6}\right) \Gamma\left(\frac{1}{3}\right)}{\Gamma\left(\frac{1}{2}\right)}-9 \frac{\Gamma\left(\frac{1}{2}\right) \Gamma\left(\frac{2}{3}\right)}{\Gamma\left(\frac{1}{6}\right)}\left(\frac{\eta}{1+\eta}\right)^{\frac{1}{3}}+\ldots\right] \\
& \approx \frac{Q^{\frac{1}{3}}}{6} \frac{2}{\pi \alpha^{\prime}} \frac{\Gamma\left(\frac{1}{6}\right) \Gamma\left(\frac{1}{3}\right)}{\Gamma\left(\frac{1}{2}\right)}-\frac{3 Q^{\frac{1}{2}}}{2}\left(\frac{2}{\pi \alpha^{\prime}}\right)^{\frac{3}{2}}\left(\frac{\Gamma\left(\frac{1}{2}\right) \Gamma\left(\frac{2}{3}\right)}{\Gamma\left(\frac{1}{6}\right)}\right)^{\frac{3}{2}} \frac{1}{S^{\frac{1}{2}}}+\ldots .
\end{aligned}
$$

The dots denote terms that are less important for $S \gg Q^{1 / 3} / \alpha^{\prime}$.

Finally, for long strings in the regime (37), the space is asymptotically AdS and we recover the GKP relation (1). 


\section{B Results for the Witten model}

The energy and the flavour charge of the Witten model can also be explicitly calculated as

$$
\begin{aligned}
E & =\frac{4 r_{0}}{2 \pi \alpha^{\prime}} \frac{\left[h\left(r_{\max }\right)\right]^{\frac{1}{2}}}{7-p} B\left(\frac{1}{2}, \frac{1}{2}\right){ }_{2} F_{1}\left(\frac{8-p}{7-p}, \frac{1}{2} ; 1 ; h\left(r_{\max }\right)\right) \\
n & =\frac{4 \omega R^{2} r_{0}}{2 \pi \alpha^{\prime}} \frac{\left[h\left(r_{\max }\right)\right]^{\frac{3}{2}}}{7-p} B\left(\frac{3}{2}, \frac{1}{2}\right){ }_{2} F_{1}\left(\frac{8-p}{7-p}, \frac{3}{2} ; 2 ; h\left(r_{\max }\right)\right)
\end{aligned}
$$

For long strings, $\omega R, h\left(r_{\max }\right) \rightarrow 1$ and we obtain

$$
E R-n=\frac{R r_{0}}{\alpha^{\prime}} \frac{2 \Gamma\left(\frac{5-p}{7-p}\right)}{(7-p) \sqrt{\pi} \Gamma\left(\frac{6-p}{7-p}\right)}-\frac{R r_{0}}{\alpha^{\prime}} \frac{(7-p) \Gamma\left(\frac{9-p}{7-p}\right)}{(5-p) \sqrt{\pi} \Gamma\left(\frac{1}{7-p}\right)}\left[1-h\left(r_{\max }\right)\right]^{\frac{5-p}{7-p}}+\ldots,
$$

which leads to (28) by virtue of (23) and (22).

\section{References}

[1] J. M. Maldacena, "The large $N$ limit of superconformal field theories and supergravity," Adv. Theor. Math. Phys. 2, 231 (1998) [Int. J. Theor. Phys. 38, 1113 (1999)] [arXiv:hepth/9711200].

[2] S. S. Gubser, I. R. Klebanov and A. M. Polyakov, "Gauge theory correlators from non-critical string theory," Phys. Lett. B 428, 105 (1998) [arXiv:hep-th/9802109].

[3] E. Witten, "Anti-de Sitter space and holography," Adv. Theor. Math. Phys. 2, 253 (1998) [arXiv:hep-th/9802150].

[4] O. Aharony, S. S. Gubser, J. M. Maldacena, H. Ooguri and Y. Oz, "Large N field theories, string theory and gravity," Phys. Rept. 323, 183 (2000) [arXiv:hep-th/9905111].

[5] A. M. Polyakov, "Gauge fields and space-time," arXiv:hep-th/0110196.

[6] S. S. Gubser, I. R. Klebanov and A. M. Polyakov, "A semi-classical limit of the gauge/string correspondence," arXiv:hep-th/0204051.

[7] D. Berenstein, J. M. Maldacena and H. Nastase, "Strings in flat space and pp waves from N = 4 super Yang Mills," JHEP 0204, 013 (2002) [arXiv:hep-th/0202021].

[8] S. Frolov and A. A. Tseytlin, "Semiclassical quantization of rotating superstring in AdS(5) x S**5," arXiv:hep-th/0204226. 
[9] J. G. Russo, "Anomalous dimensions in gauge theories from rotating strings in $A d S_{5} \times$ $S^{5}, "$ arXiv:hep-th/0205244.

[10] A. Armoni, J. L. Barbón and A. C. Petkou, "Orbiting strings in AdS black holes and $\mathrm{N}=4 \mathrm{SYM}$ at finite temperature," arXiv:hep-th/0205280.

[11] G. Mandal, N. V. Suryanarayana and S. R. Wadia, "Aspects of semiclassical strings in AdS(5)," arXiv:hep-th/0206103.

[12] M. Alishahiha and M. Ghasemkhani, "Orbiting membranes in M-theory on AdS(7) x S**4 background," arXiv:hep-th/0206237.

[13] J. A. Minahan, "Circular semiclassical string solutions on $\operatorname{AdS}(5) \times \mathrm{S}^{* *} 5$," arXiv:hepth/0209047.

[14] H. J. de Vega, A. L. Larsen and N. Sanchez, "Semiclassical quantization of circular strings in de Sitter and anti-de Sitter space-times," Phys. Rev. D 51, 6917 (1995) [arXiv:hep-th/9410219].

[15] D. J. Gross and F. Wilczek, "Asymptotically Free Gauge Theories. 2," Phys. Rev. D 9, 980 (1974).

[16] E. G. Floratos, D. A. Ross and C. T. Sachrajda, "Higher Order Effects In Asymptotically Free Gauge Theories. 2. Flavor Singlet Wilson Operators And Coefficient Functions," Nucl. Phys. B 152, 493 (1979).

[17] A. González-Arroyo and C. López, "Second Order Contributions To The Structure Functions In Deep Inelastic Scattering. 3. The Singlet Case," Nucl. Phys. B 166, 429 (1980).

[18] H. J. de Vega and I. L. Egusquiza, "Planetoid String Solutions in $3+1$ Axisymmetric Spacetimes," Phys. Rev. D 54, 7513 (1996) [arXiv:hep-th/9607056].

[19] S. Kar and S. Mahapatra, "Planetoid strings: Solutions and perturbations," Class. Quant. Grav. 15, 1421 (1998) [arXiv:hep-th/9701173].

[20] A. Tseytlin at "strings 2002", http://www.damtp.cam.ac.uk/strings02/avt/tseytlin/; see also, "Semiclassical quantization of superstrings: $A d S_{5} x S^{5}$ and beyond," arXiv:hepth/0209116.

[21] E. Witten, "Anti-de Sitter space, thermal phase transition, and confinement in gauge theories," Adv. Theor. Math. Phys. 2, 505 (1998) [arXiv:hep-th/9803131]. 
[22] D. J. Gross and H. Ooguri, "Aspects of Large N Gauge Theory Dynamics as Seen from String Theory," Phys. Rev. D58, 106002 (1998) [arXiv:hep-th/9805129].

[23] J. M. Maldacena, "Wilson loops in large N field theories," Phys. Rev. Lett. 80, 4859 (1998) [arXiv:hep-th/9803002].

[24] S. J. Rey and J. Yee, "Macroscopic strings as heavy quarks in large N gauge theory and anti-de Sitter supergravity," Eur. Phys. J. C 22, 379 (2001) [arXiv:hep-th/9803001].

[25] Y. Kinar, E. Schreiber and J. Sonnenschein, "Q anti-Q potential from strings in curved spacetime: Classical results," Nucl. Phys. B 566, 103 (2000) [arXiv:hep-th/9811192].

[26] A. Chamblin, R. Emparan, C. V. Johnson and R. C. Myers, "Charged AdS black holes and catastrophic holography," Phys. Rev. D 60, 064018 (1999) [arXiv:hep-th/9902170].

[27] N. Itzhaki, J. M. Maldacena, J. Sonnenschein and S. Yankielowicz, "Supergravity and the Large N Limit of Theories with Sixteen Supercharges," Phys. Rev. D58, 046004 (1998) [arXiv:hep-th/9802042]. 